\title{
fo América Latina: ¿Una región atractiva en momentos de crisis?
}

\author{
Miguel A. Lazo
}

Universidad para la Cooperación Internacional, Costa Rica

Resumen: El principal objetivo de este artículo ha sido identificar las principales oportunidades de crecimiento existentes en la región de América Latina, tomando en consideración los momentos de crisis de los que se ha sido testigo en los últimos años. El campo de interés está centrado en identificar las restricciones en el crecimiento económico con que cuenta la región, con el fin de determinar las de mayor relevancia. Con base al análisis de estas restricciones, el autor evalúa los escenarios y presenta los principales retos y oportunidades que se tienen, dando respuesta a la interrogante planteada. Los resultados demuestran su enorme potencial, así como los principales desafíos que deben ser superados. Indudablemente, el momento que vivimos en la economía mundial es sumamente delicado, por lo que los esfuerzos deben estar destinados a superar los retos que se tienen como región, y lograr a través de las fortalezas con que se cuenta aprovechar este enorme potencial. Cabe destacar el papel que juega el sector servicios dentro de la estructura económica, el rol de la infraestructura y su incidencia en el desarrollo de la región, así como el papel crucial que juega el comercio internacional como motor de la economía. Los datos aportados reflejan las fortalezas de la región, pero éstas se han visto mermadas por distintos elementos en los últimos años, como la reciente caída de uno de sus principales mercados emergentes como lo es Brasil, quien lucha por alzar el vuelo. Indudablemente, hay muchos factores en juego pero el reto está en consolidar nuevamente a la región como un destino atractivo para las demás economías.

Abstract: The main objective of this article has been to identify the principal growth opportunities in Latin America, considering the crisis of the last years. The main idea is to identify the restrictions of growth economic in the region and to determine the most important ones. With this analysis, the author evaluates the scenarios and shows the principal challenges and opportunities, giving an answer to the main question of this article. The results demonstrate the enormous potential and the principal challenges to overcome. Certainly, the actual situation in the worldwide economy is fragile; for that reason, the efforts should be aimed at overcoming challenges as a region, and achieving this through its strengths 
and its big potential. It's important to mention the role of the service sector inside of the economic structure, the role of the infrastructure and its relevance in the development of the region and the critical role of the commerce as the engine of the economy. The data provided reflects the strengths of the region; but they have been reduced for different elements in recent years, as the recent fall in one of its principal emerging markets such as Brazil is struggling to take off. Undoubtedly, there are many factors at play, but the challenge is to consolidate the region as an attractive destination again for other economies.

Palabras claves: América Latina, restricciones, oportunidades, crecimiento económico, crisis

Keywords: Latin America, restrictions on growth, opportunities, economic growth, crisis

\section{Introducción}

La economía mundial está en busca de un punto de equilibrio después de que ha sido testigo de distintas crisis que han traído consigo un panorama brumoso para muchas naciones y regiones, y para sus economías. Desde la crisis mundial que inició en el año 2007 con la quiebra de algunos pequeños bancos de menor inversión, y que terminó estallando en 2008 con la denominada "burbuja inmobiliaria" que ocasionó el debacle del sistema financiero estadounidense, pasando por la crisis financiera del 2012 ocasionada por el colapso de las finanzas públicas de varios países, la ralentización del crecimiento económico de las economías más grandes como la de EUA, la Zona del Euro y la de Japón, la incertidumbre acerca del euro y su repercusión en los mercados financieros internacionales, esto según datos brindados por el Fondo Monetario Internacional ${ }^{1}(2014 a)$; para luego ser testigo, posteriormente, de la caída de los precios del petróleo que ha traído consigo ganadores y perdedores en ese ajuste de precios, incluidos diversos países de América Latina que dependen en gran medida del "oro negro" como Venezuela, Ecuador, Colombia, México y Brasil; hasta la reciente crisis del gigante asiático chino que durante años ha sido el motor de la economía a nivel mundial pero que desde hace un tiempo se ha venido desacelerando, pasando por alto las distintas alarmas que se le han presentado, y nuevamente ha traído el temor a nivel mundial de si estamos o no a las puertas de una nueva crisis global. Este escenario trae a la luz la incertidumbre de si la región de América Latina puede verse favorecida y aprovechar esta 
diversidad de elementos, y más si evaluamos que durante años el crecimiento económico ha sido lento $\mathrm{y}$, por ende, relativamente bajo; situación que puede ser constatada si se compara con el de otras regiones que en condiciones similares y en menor tiempo han logrado mejorar sus estadísticas económicas, sociales e indicadores, así como las condiciones de vida de sus habitantes. Un ejemplo claro es el crecimiento promedio del Producto Interno Bruto (PIB) per cápita de la región en la década del 2000 que fue del 1.6\% anual, inferior al de otras regiones como la del Este de Asia y Pacífico que fue del $3 \%$. Este escenario refleja, en un primer momento, una situación crítica para América Latina porque la práctica demuestra que solamente mediante un crecimiento elevado y sostenido los países pueden dar un salto de calidad significativo en sus niveles de desarrollo. Un caso emblemático sobre este punto es Corea del Sur, quien desde la década de los 60 ha tenido incrementos sustanciales de su economía en diversos períodos de tiempo, los cuales si los comparamos con las estadísticas de los países de la región con mejor desempeño como Chile, lo supera con creces (Corea del Sur tiene un PIB per cápita mayor al de todos los países de la región y su crecimiento es considerado una de las grandes historias de éxito del último siglo, siendo catalogada como una de las potencias económicas a nivel mundial con una sólida base industrial, y es uno de los Estados claves dentro de su región en la actualidad; razón por la cual ha sido objeto de diversos estudios que han tenido como finalidad identificar los factores claves dentro de su crecimiento económico y desarrollo social), y más aún si se toma en consideración que durante la Segunda Guerra Mundial fue ocupada por la antigua Unión Soviética y los Estados Unidos de América (EUA), y que en años posteriores sufrió el embate de una guerra que finalizó en 1953 con las negociaciones de paz y la división definitiva de las dos Coreas; lo cual trajo como consecuencia que el país estuviera ubicado entre los más pobres del mundo en ese tiempo. Definitivamente, este ejemplo logra dar validez a esta teoría, ya que ha permitido mediante esta dinámica de crecimiento que estos países puedan reducir las brechas existentes con los países desarrollados como EUA o China, destacando que dicho crecimiento ha venido acompañado obligatoriamente de una serie de reformas, ajustes y cambios estructurales radicales que permitieron esa dinámica, pero que demuestra los impresionantes resultados que se pueden lograr si se logra trabajar de manera coordinada. 


\section{Gráfico 1. Crecimiento del PIB\% por regiones entre 1965-2014}

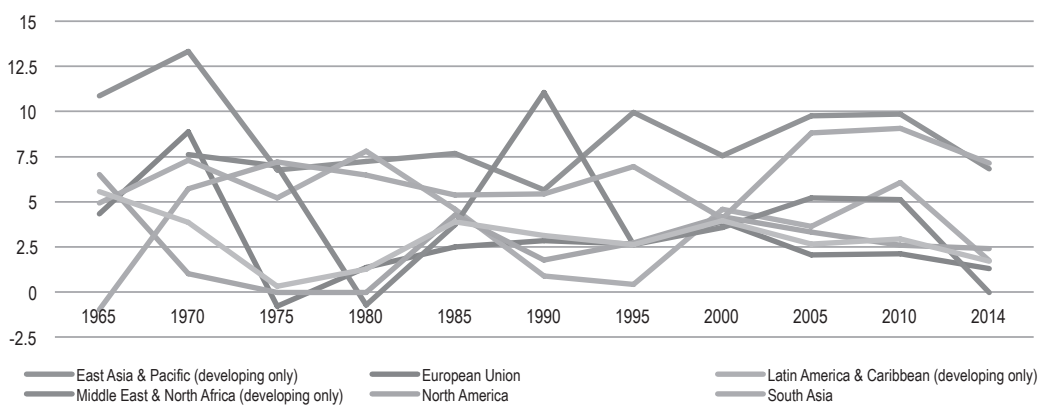

Fuente: Datos obtenidos de las estadísticas anuales del Banco Mundial, 2016.

\section{Restricciones al crecimiento de América Latina}

Al momento de evaluar la situación de la región, diferentes instituciones han tratado de descifrar los orígenes de la problemática existente en relación al crecimiento económico y han analizado profundamente muchas de las restricciones con las que nuestra región cuenta, logrando identificar factores internos y externos importantes. En el caso de los factores externos - de los que ya se ha hecho mención están las diferentes crisis que han afectado a todos los países en su mayoría y sin distinción, y que han dado poco margen de maniobra a los países latinoamericanos y sus políticas estatales. En el caso de los factores internos, se han logrado identificar las principales restricciones entre las que están los bajos niveles de inversión privada, el bajo acceso al financiamiento, los bajos retornos sociales, las fallas de gobierno y de mercado, la mala infraestructura, la pobre integración, entre muchos otros elementos, situación que se complica si se considera que algunas de estas restricciones datan de muchos años, mientras que otras son recientes y han venido a acrecentar el problema (Agosín, Fernández-Arias \& Jaramillo, 2009). Es necesario recalcar que estas restricciones se presentan en muchos de los países, y en cada país se padecen muchas de estas restricciones que tienden a cambiar a lo largo del tiempo atendiendo a la situación y a las condiciones del país. La restricción que se ha visto presente en la mayoría de las economías de la región mediante la figura de instituciones débiles son las fallas de gobierno, las que junto con el crimen, la protección ineficiente de los derechos de propiedad hasta la implementación de políticas económicas débiles o ineficaces, conforman la categoría y brindan un panorama del origen del problema y del enorme trabajo 
que se tiene si se desea mejorar la situación de la región; en este punto es de considerar el riesgo político que esto ocasiona y que ha reaparecido con fuerza, ya que es uno de los principales temores que se ha ocasionado por esta inestabilidad política que se vive o que es percibida en muchos países de la región, a tal punto que se ha llegado a considerar que en ninguna otra región del mundo existe en los negocios un riesgo político tan alto como en la región (Bringas, 2013). Esto no quiere decir que estos países sean los más inestables porque evidentemente hay otros países mucho más inestables políticamente, pero los inversionistas ya están conscientes de éstos y no se atreven a incursionar porque son países que por tradición han tenido poca inversión, como puede ser el caso de Somalia.

\section{Gráfico 2. Árbol de decisiones para examinar los obstáculos a la inversión privada}

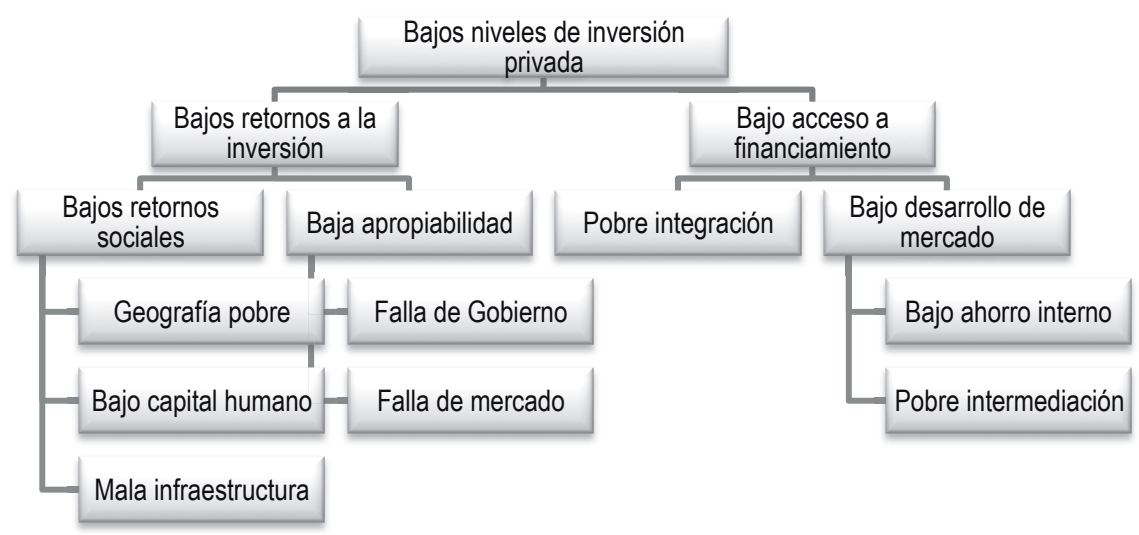

Fuente: Datos obtenidos de Hausmann, Hwang \& Rodrik (2005).

Un caso que se hace necesario mencionar, considerando la relevancia y el aporte que brinda directamente, es el de Brasil, la principal economía de la región y una de las principales economías a nivel global y parte del grupo de economías emergentes denominado BRICS (Brasil, Rusia, India, China y Sudáfrica), que previsiblemente marcarán el devenir político y económico de este siglo XXI y que comparten entre sí las cualidades de tener extensiones territoriales vastas, así como riquezas y recursos naturales en abundancia con una enorme población en conjunto y cifras de crecimiento llamativas a pesar de la crisis en diversos lapsos temporales, pero que en los últimos años ha visto mermado su desempeño, llegando a presentar niveles decepcionantes si se les compara con el de períodos pasados. Dichos 
datos se han visto reflejados en sus distintos indicadores que muestran el debilitamiento de su economía donde una combinación de factores externos e internos la han afectado severamente. La aceleración de su economía en la década pasada se debió en gran medida a la recuperación de su demanda, incremento de sus exportaciones, una mayor inversión pública y a reformas regulatorias que permitieron incrementar el ingreso, y que junto con una política fiscal y monetaria previsible sirvieron de base para su crecimiento. Por otra parte, su decaimiento en los últimos años ha sido ocasionado por el deterioro de los ingresos fiscales y las políticas públicas, el aumento de su deuda externa que tuvo un fuerte incremento al pasar de US $\$ 240,495$ millones en 2007 (17.2\% del PIB) a US\$556,245 millones en 2014 (23.6\% del PIB), mercados accionarios con comportamientos volátiles, una declinante competitividad y las perturbaciones políticas, entre otros elementos según datos del Instituto Provincial de Estadísticas y Censos ${ }^{1}$ (2015). Por consiguiente, el reto es inmenso ya que entre las condiciones necesarias para que el país vuelva a crecer está el ajuste de su política económica, por lo que estará a la vista del ojo público en su desarrollo.

\section{El sector de servicios dentro de la estructura económica}

Se ha podido observar que existen muchas restricciones para el crecimiento de América Latina tanto internas como externas y que el bajo rendimiento que ha presentado en diferentes apartados no se debe solamente a uno de esos factores sino que, en todos los casos, se debe una combinación de muchos de ellos. Sin embargo, la principal restricción con la que se cuenta es la baja productividad que ha sido catalogada como una de las determinantes más importantes de la desaceleración de la región desde la década de los 80 (Ros, 2011), por lo que identificar las causas que la originan se convierte en una necesidad para proponer planes de acción que permitan mejorar los niveles de productividad, considerando que al mejorar este elemento habría más inversión y educación con lo que se aceleraría el crecimiento anhelado, y se reduciría la brecha existente con los países en desarrollo y/o emergentes. Esta necesidad trae consigo el enfoque inmediato que se le debe dar a los planes para mejorar la productividad. Por tradición, la mayor parte de los países latinoamericanos han basado su matriz productiva en el sector agropecuario, ejemplo de ello son países como El Salvador, el cual durante buena parte del siglo pasado basó su economía en productos primarios considerados estrella como el café, el azúcar e incluso en su momento; el añil 
(producto que decayó con la salida al mercado de los tintes artificiales). Posteriormente, la región -con el estallido de la tendencia de libre comercio a nivel mundial en la década de los 90- se dedicó a preparar un escenario que permitiera aprovechar este movimiento para abrir los mercados, implementando políticas de apertura comercial que permitieran este objetivo sin considerar la estructura productiva de cada uno de sus países. Este último escenario permitió cambios sustanciales en la producción y en la oferta exportadora, razón por la cual muchos de los esfuerzos por mejorar la productividad se enfocaron en el sector industrial, como muestra de ello se tienen las enormes inversiones en maquilas, parques de servicios y parques industriales. En los últimos años, la tendencia a nivel mundial ha cambiado y ha surgido el sector de servicios que desde la década de los 80 se ha consolidado, presentando incrementos sustanciales, sobrepasando incluso al sector de bienes que por décadas había sido el sector por excelencia y, hasta cierto punto, el sostén de muchas de las economías latinoamericanas. Tal ha sido su incremento que representa en la actualidad el $20 \%$ del comercio mundial, dando una pauta de donde se deben dirigir los esfuerzos para elevar los niveles de productividad. Un punto clave dentro de su entorno es que no solamente se logra que buena parte del recurso humano se dedique a estas actividades, sino que adicionalmente se contribuye a que su desarrollo sea sostenible, permitiendo el crecimiento indirecto de otros sectores como el industrial o agropecuario, al verse inmerso mediante muchas de sus actividades como la de transporte, comunicaciones y logística, por mencionar algunas de ellas. Según estimaciones de la Organización Mundial del Comercio ${ }^{2}$ (2014), en su informe anual de estadísticas se hacía énfasis en el crecimiento de este rubro que solamente en el 2013 había ascendido a US\$4,645 billones y que representaba un incremento del $6 \%$ en el total global, presentándose los mayores incrementos en la Comunidad de Estados Independientes (CEI) con un $9 \%$, los países europeos con un $7 \%$ y en los países de América del Norte y la región del Este Asiático con un $5 \%$ que obedeció en gran medida a la recuperación del comercio europeo después de la crisis de la Zona Euro y a la recuperación de Estados Unidos, aunque su incremento significativo en las últimas décadas obedece a la aparición de las economías en desarrollo con mayor dinamismo económico como los países asiáticos. La oportunidad latente para la región de América Latina dentro de dicho sector se debe en gran medida a que estas actividades en cierto momento eran operadas por multinacionales de los países desarrollados pero que se han ido externalizando y ubicando en países emergentes en su afán por reducir los costos, así como por 
la revolución en las tecnologías de información y la liberalización del comercio, y aunque la región ha ingresado recientemente en el juego porque en un inicio países como la India, los asiáticos y algunos de Europa del Este fueron el polo del crecimiento. En los últimos años, este sector se ha vuelto el gran empleador de la región por su capacidad de absorber trabajadores calificados, por lo que los esfuerzos se han dirigido a convertir a la región en un exportador de servicios, destinando sus acciones en atraer inversiones de alto valor agregado, ejemplo de ello es Chile, quien junto con Brasil, Argentina y México han prestado especial atención a los sectores de turismo, servicios médicos, inteligencia artificial, cuidados personales, informática, tecnologías de información, servicios financieros, entre otros.

\section{Gráficos 3 y 4. Exportaciones de servicios comerciales, 2005-2013}

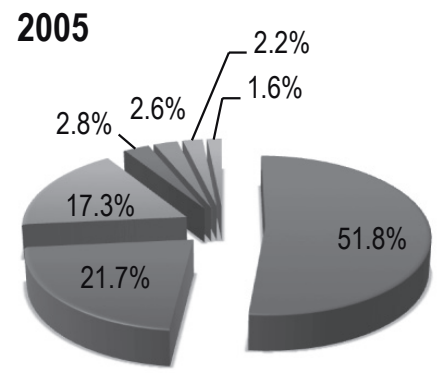

\section{3}

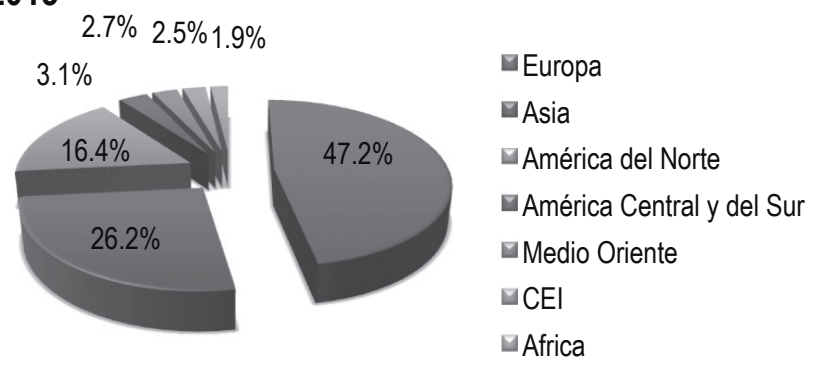

Fuente: Datos obtenidos de las estadísticas anuales de la OMC, 2016. 


\section{El rol de la infraestructura}

Como se ha podido observar, los países de América Latina presentan una serie de restricciones para lograr un desarrollo económico sostenible. En este apartado, la Comisión Económica para América Latina y El Caribe 3 (2015) ha presentado hallazgos relacionados a la desaceleración económica en la región y su relación con la inversión donde ha determinado que la caída de la tasa de inversión y la menor contribución de formación bruta de capital al crecimiento son preocupantes por su incidencia en el ciclo económico y en el crecimiento, y donde se ha logrado determinar un concepto de infraestructura más amplio e inclusivo, vinculado al crecimiento, productividad y al desarrollo territorial e institucional. Por otra parte, si se considera que en los últimos años ha sido recurrente constatar la relevancia de la infraestructura en la vida de las personas, implica que éste tiene una relevancia crucial para los países y sus gobiernos. En un primer momento es de hacer mención de la importancia político-estratégica adquirida, puesto que con una adecuada infraestructura los países indudablemente podrán insertarse de manera eficiente en los escenarios mundiales, el siguiente punto es la determinación de cuál es el tipo de infraestructura que se necesita para lograr dichos fines, y por último; la correcta forma de financiamiento de los mismos (Rozas, Bonifaz y
Guerra García, 2012). Lo dicho anteriormente implica que transitar de una manera eficiente en ese camino requiere de un cambio estructural que reoriente las políticas hacia una fuerte inversión, ya que solamente de esa manera se logrará asegurar el crecimiento, productividad y sostenibilidad deseados. En este aspecto, América Latina presenta un rezago significativo al no haber alcanzado los niveles deseados que el Banco Mundial estima convenientes para sustentar las expectativas de crecimiento en la región y que oscilan entre el $4-5 \%$, situación que es descrita por el FMl (2014b) en su Informe Anual de Competitividad donde se manifiesta la situación existente en la región (la infraestructura es uno de los pilares que conforman el índice de competitividad) y hace énfasis en la falta de inversión en esta área comparado con el de otras regiones o países donde se destinan altos porcentajes del PIB del país para su desarrollo; esto constituye un obstáculo en el esfuerzo por mejorar sus índices de crecimiento e implementación de políticas de desarrollo social. Si en esta dinámica se compara a los países de la región con respecto a otros países o regiones del mundo, se podrá notar que existen países que están invirtiendo menos que antes y menos que muchas economías emergentes. Relacionado a este último punto se considera que la región solamente invierte 
aproximadamente el $3 \%$ de su PIB, muy lejos del $5 \%$ propuesto para cerrar la brecha existente actualmente y que comparado a lo que se invierte en la región de Asia donde se destina aproximadamente un $6 \%$, o incluso del $8-10 \%$ en algunos de dichos países en este rubro denota una importante diferencia (Corporación Andina de Fomento, 2015). Esta disminución ha sido ocasionada en gran medida por la caída de la inversión pública en infraestructura y que no pudo ser compensada con la inversión privada en ese rubro, pero es de mencionar que no se debe tratar solamente de mejorar los niveles de inversión sino también de definir cuáles son los proyectos en infraestructura que se requieren para lograr ventajas competitivas mediante la modernización de los puertos, aeropuertos, fronteras, entre otros, de tener claras las proyecciones para el futuro, y hacer adecuados ajustes y proyectos que permitan determinar las necesidades para los próximos años. Es de recordar que la región debe de combatir con otra serie de elementos en este género; los avances diferenciados en los sistemas de producción ocasionados en la década de los 90 por los avances en la producción de commodities, los problemas para intentar satisfacer la creciente actividad industrial debido a los flujos logísticos y a los déficits en la producción de energía o el crecimiento diferenciado entre los sectores que conforman el rubro (el sector de transporte no ha crecido a un ritmo similar al que ha crecido el sector de las telecomunicaciones o energético), así como con relación a las necesidades de su crecimiento económico, las demandas sociales y las posibilidades comerciales y de negocios que ofrecen la integración regional y el mercado mundial (Sistema Económico Latinoamericano y del Caribe (SELA), 2011), por ende, los retos venideros en este rubro son cruciales y están encaminados a mejorar la calidad y cobertura de los servicios de infraestructura. Sin embargo, cabe destacar que en los últimos años, y a pesar de las deficiencias demostradas, se han efectuado esfuerzos enormes por mejorar esta situación, por tal razón desde la década de los 90 se han presentado importantes avances como el acceso competitivo en varios servicios (comunicaciones, gas, tecnología, entre otros.), el desarrollo de redes de agua potable y saneamiento, la creación de empresas mixtas, la ampliación de redes ferroviarias, la modernización de terminales portuarias, la mejora de las vías navegables, las redes de transporte por carretera, entre otros proyectos. Por lo que actualmente, el rubro se encuentra en un período de expansión en su uso y provisión. Un aspecto que es necesario mencionar es que los proyectos de infraestructura en general tienen como característica en común los costos de capital elevados en sus inicios, razón por la cual muchos solamente han quedado en papel y no han 
podido ser ejecutados al sobrepasar las capacidades del Gobierno y no haber encontrado un proveedor privado para su financiamiento. Por lo que en un inicio, el reto está en encontrar las fuentes de financiamiento, y es en este punto cuando surgen las Asociaciones PúblicoPrivadas (APP), que han demostrado ser un importante instrumento a la hora de invertir recursos privados en proyectos de infraestructura con potencial de incorporar tecnología, innovación, eficiencia y calidad en la provisión del sector público, siempre y cuando hayan sido correctamente utilizados, puesto que caso contrario, son capaces de comprometer la sostenibilidad de las finanzas públicas y asumir obligaciones fiscales futuras (Cipotella Tomassian, 2015). La experiencia en APP ha dejado importantes lecciones, considerando que los resultados observados en la participación activa del sector privado en el desarrollo de la infraestructura han sido increíbles desde 1990, porque buena parte de las inversiones destinadas han sido captadas por la región (se estima que la región ha acumulado cerca de 2,045 proyectos de infraestructura con participación privada con una inversión total de US $\$ 958,746$ millones, según datos obtenidos del Banco Mundial, 2016); sin embargo, a pesar de los incrementos, no se ha podido compensar la caída de la inversión pública en diversos períodos de este lapso (Vassallo Magro, 2015); destacando a su vez que la distribución de este tipo de proyectos se ha concentrado en seis países (Brasil, México, Argentina, Chile, Colombia y Perú).

\section{Gráfico 5. Número de proyectos de inversión privada por región, 1990-H1 2015}

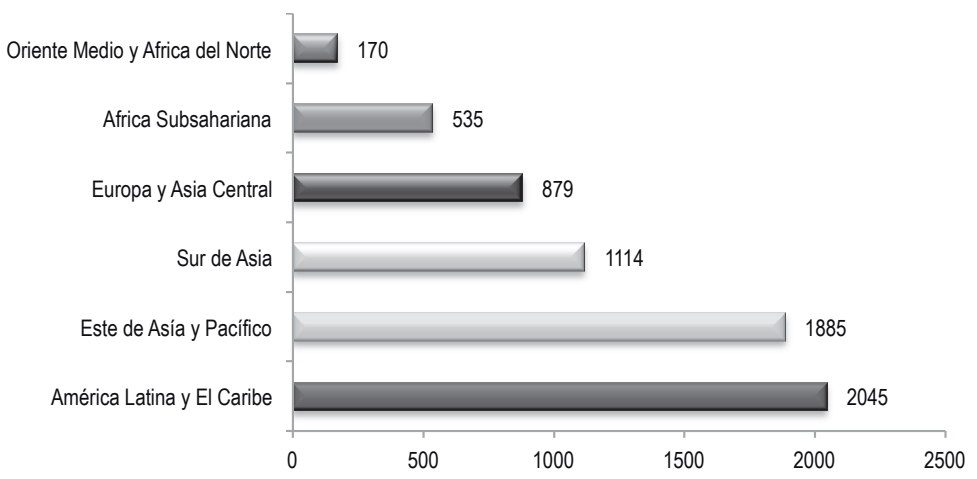

Fuente: Datos obtenidos de PPI (Private Participation in Infrastructure) del Banco Mundial, 2016. 
El comercio internacional como motor del crecimiento

América Latina ha tenido una fuerte expansión comercial en los últimos años, producto de la tendencia en comercial de sus miembros -en la década de los 90 , la mayor parte de los países de la región implementaron políticas de apertura comercial-, sin embargo, ha quedado claro que este fenómeno no garantiza un desarrollo o crecimiento económico sostenido y que puede constatarse al evaluar el crecimiento medio de la región que ha estado por debajo de la media de los países de la Organización para la Cooperación y Desarrollo Económico (OCDE) en los últimos años, o en el desarrollo social que se busca en la mayor parte de los casos (OCDE/CEPAL/CAF, 2015). La globalización y sus elementos son procesos que datan de muchos años, los que se han profundizado y diversificado, y donde ha quedado demostrado que para lograr resultados satisfactorios la economía de un país debe estar abierta al comercio y a la inversión con el resto del mundo, porque ningún país ha logrado mejoras significativas en su economía, y en el nivel de vida de sus habitantes, sin estar inmerso en este tejido; sólo es de lanzar una mirada a los países asiáticos y a los niveles de desarrollo que han presentado. En el contexto global, los bienes primarios han perdido participación, dando paso a productos manufacturados con un mayor nivel de elaboración. Sin embargo, la región latinoamericana aún depende en gran medida de la exportación de productos primarios, situación que es comprensible si se considera la enorme abundancia de recursos naturales comparado con el de otras regiones, aunque es de recordar que durante los primeros años del proceso de liberalización en la región se vino consigo un proceso de reestructuración de las economías donde algunas se enfocaron en sus productos primarios, otras se especializaron en la exportación de productos manufacturados (auge de las maquilas), mientras que otras buscaron una vía intermedia para modificar su matriz productiva (Buitrago, 2009). En este punto, decir que hay que olvidar este potencial con que se cuenta es ilógico; por lo que la estrategia debe ir dirigida a convertir los productos primarios en el eje transversal de la inserción de la economía en el mercado global y en el elemento principal para la diversificación de la producción hacia sectores especializados. Por otra parte, esta apertura comercial en los últimos años ha llamado la atención de un socio comercial para la región: China, quien es un elemento de cambio real en el entorno mundial y que forma parte también del grupo BRICS. La relación entre China con la región ha experimentado una expansión significativa en los últimos 15 años, durante los cuales se ha multiplicado 22 veces, y ha puesto en evidencia las deficiencias 
estructurales de un crecimiento basado en las materias primas como lo ha tenido la región que -junto con la menor demanda china en los últimos años- ha mostrado diferentes patrones de desaceleración. Sin embargo, al haber quedado al descubierto estas diferencias sustanciales, los gobiernos han iniciado la búsqueda de medios que permitan a la región seguir siendo competitiva y atractiva para China, evaluando políticas de desarrollo productivo innovadoras que permitan obtener ventajas competitivas y modificar de ser posible, la matriz productiva con que se cuenta. De igual manera, ha sido evidente la necesidad de inversión en temas como capacitación, integración e infraestructura que se requiere en muchas áreas; así como la necesidad de mejorar las regulaciones, la capacidad de los gobiernos en proyectos financiables, la sostenibilidad ambiental y la transparencia en temas de política (OCDE/CEPAL/CAF, 2015). No cabe duda que el cambio ya se ha iniciado, y existe un gran reto en materia comercial para la región si desea verse beneficiada de esta relación, donde debe incorporar dentro de sus estrategias la ampliación de objetivos, la diversificación y la cooperación interregional y extrarregional entre los países, porque a medida que aumente la participación de China en nuevas plataformas comerciales y de desarrollo es necesario que los países de la región tengan lazos más fuertes y un mayor grado de compromiso con sus vecinos. Una tarea nada envidiable y que debe ir de la mano con las demás áreas.

\section{Gráfico 6. Crecimiento del volumen del comercio mundial de mercancías por regiones, 2005-2013}

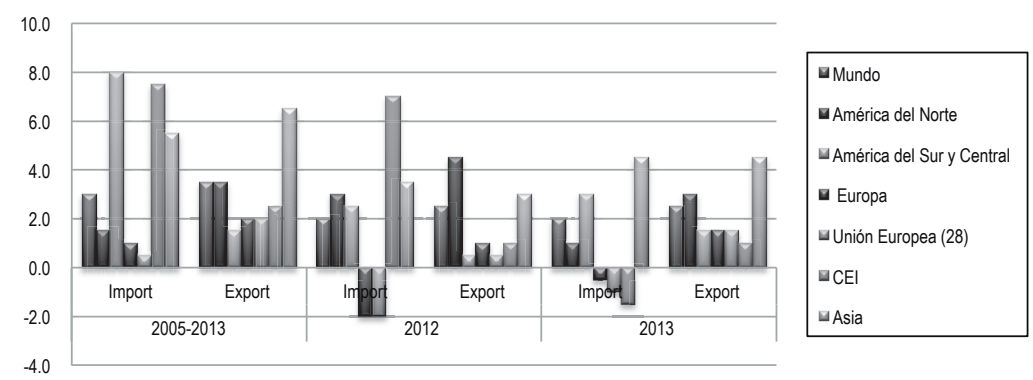

Fuente: Datos obtenidos de las estadísticas anuales de la OMC, 2016. 


\section{Conclusiones}

Los resultados de este análisis permiten extraer conclusiones que se formulan seguidamente y que podrán ser contrastadas en estudios más complejos que contemplen mayor representatividad de los datos:

América Latina tiene una historia de inestabilidad y de crisis económicas que datan de muchas décadas y que le han marcado durante diversos períodos, lo que ha ocasionado que la región haya perdido competitividad con otras regiones o países que en menos tiempo y con mayores dificultades han logrado salir adelante. La desaceleración económica que se ha visto reflejada es sinónimo del clima de inestabilidad que se vive y que se fortalece con una serie de restricciones internas y externas que hacen más complicado este panorama. Brasil es el ejemplo idóneo, porque en los últimos años ha pasado de ser una nación emergente con un enorme potencial a presentar serios problemas en su economía, producto de la conjunción de varias restricciones.

La baja productividad que se tiene, y que es una de las principales restricciones de los países de la región al repetirse en diversos países y escenarios, hace necesario el desarrollo de mecanismos y estrategias para modificar la matriz productiva con que se cuenta, esto con la finalidad de fortalecer aquellos sectores que en los últimos años han presentado incrementos significativos y que poseen un gran potencial, tal como el sector de servicios. La meta principal debe ser en este sentido: cambiar la dependencia de las exportaciones de productos primarios por la de productos especializados y de servicios.

En gran medida, la desaceleración de América Latina se debe a la baja inversión de la que se ha sido testigo, por consiguiente, la situación en la que se encuentra no es del todo ajena a los diversos actores, y más si se evalúa que la región ha experimentado caídas en la inversión pública que no han podido ser compensadas por las inyecciones de capital de la inversión privada. Sin embargo, es conocida la relación existente entre desarrollo, crecimiento, inversión e infraestructura, por tal razón, se hace necesario la implementación de planes de acción que permitan mejorar la calidad de la infraestructura con la que se cuenta, lo que implica un cambio sustancial que reoriente las políticas hacia la inversión. Surgiendo en este punto la necesidad de buscar medios de financiamiento que permitan mejorar estos niveles de inversión, y donde las APP aparezcan como una de las opciones viables, siempre y cuando se implementen adecuadamente de parte de los 
gobiernos, quienes deben hacer el esfuerzo por desarrollar antes de su puesta en ejecución un correcto diseño, regulación y control.

Por último, la globalización es un proceso que data de hace muchas décadas, por lo tanto, tratar que una nación o región salga adelante sin ser parte del mismo es imposible. En ese sentido, la región se ha visto inmersa en un proceso de apertura comercial desde la década de los 90 que ha traído consigo la relación comercial con diversos países, siendo uno de ellos China, quien ostenta un lugar privilegiado en la economía mundial. Por consiguiente, los esfuerzos de países ahora están encaminados a aprovechar la relación con el gigante asiático, donde ya se ha iniciado la búsqueda de medios que sigan haciendo atractiva a la región. Es importante recalcar que parte de este proceso implica modificar la matriz productiva con que se cuenta -sobre todo si consideramos que la región ha dependido por tradición de la exportación de productos primarios-y prestar atención a aquellos sectores que han incrementado sus exportaciones en los últimos años. Este proceso se considera sumamente beneficioso porque en el afán por mejorar las condiciones con el gigante asiático se logrará establecer condiciones ideales con los demás socios comerciales de la región.

\section{Referencias}

- Agosín, M., Fernández Arias, E. \& Jaramillo, F. (2009). Growing pains: binding constraints to productive investments in Latin America. Washington D.C.: Banco Interamericano de Desarrollo.

- Bringas, R. (2013). Negocios y política en América Latina: El factor político como factor impoderable para los inversionistas. European Scientific Journal. Recuperado de http://eujournal.org/index.php/esj/article/ download/773/807

- Buitrago, R. (2009). Reformas comerciales (apertura) en América latina: revisando sus impactos en el crecimiento y el desarrollo. Bogotá, Colombia: Universidad de La Salle. Recuperado de http://www.scielo.org.co/pdf/rfce/ v17n2/v17n2a07.pdf

- Cipotella Tomassian, G. (2015). Financiamiento de la infraestructura para la integración regional. Alternativas para América del Sur. Santiago, Chile: Publicación de las Naciones Unidas. Recuperado de http://repositorio. cepal.org/bitstream/handle/11362/39839/S1501366_es.pdf?sequence=1

- Corporación Andina de Fomento (CAF). (2015). IDEAL 2014. Infraestructura en el desarrollo de América Latina 2014: Resumen ejecutivo. Bogotá, Colombia: Editorial CAF. Recuperado de http://scioteca.caf. 
com/bitstream/handle/123456789/697/IDEAL_Resumen_Ejecutivo. pdf? sequence $=8$ \&isAllowed $=y$

- Estudio económico de América Latina y El Caribe (CEPAL). (2015). Desafíos para impulsar el ciclo de inversión con miras a reactivar el crecimiento. Santiago de Chile, Chile: Publicaciones de las Naciones Unidas. Recuperado de http://repositorio.cepal.org/bitstream/handle/11362/38713/ S1500733_es.pdf?sequence $=106$

- FMI. (2014a). Global Financial Stability Report. Washington D.C., Estados Unidos: IMF Publications Services. Recuperado de http://www.imf.org/ External/pubs/FT/GFSR/2014/01/pdf/text.pdf

- FMI. (2014b). Global Competitiviness Report. Washington D.C., Estados Unidos: IMF Publications Services. Recuperado de http://www3.weforum. org/docs/WEF_GlobalCompetitivenessReport_2014-15.pdf

- Hausmann, R., Hwang, J. \& Rodrik, D. (2005). What you export matters (Documento de trabajo). John F. Kennedy School of Government, Harvard University. Cambridge.

- IPEC. (2015). La economía de Brasil: Presente futuro. Santa Fe, Argentina. Gobierno de Santa Fe. Recuperado de https://www.santafe.gov. ar/index.php/web/content/download/219987/1145933/version/1/ file/2015+julio+20+Brasil+hr.pdf

- OCDE/CEPAL/CAF. (2015). Perspectivas económicas de América Latina 2016: Hacia una nueva asociación con China. OECD Publishing, Paris. Recuperado de http://dx.doi.org/10.1787/9789264246348-es

- OMC. (2014). Estadísticas del comercio Internacional. Ginebra, Suiza: Publicaciones de la OMC. Recuperado de https://www.wto.org/spanish/res_s/ statis_s/its2014_s/its2014_s.pdf

- Ros, J. (2011). La desaceleración de la productividad en América Latina: dos interpretaciones vol. 8, № 23. México D.F.: Economía, UNAM. Recuperado de http://www.economia.unam.mx/publicaciones/nueva/ econunam/23/02ros.pdf

- Rozas, P., Bonifaz, J. \& Guerra García, G. (2012). El financiamiento de la infraestructura. Propuestas para el desarrollo sostenible de una política sectorial. Santiago, Chile: Publicación de las Naciones Unidas. Recuperado de http://repositorio.cepal.org/bitstream/handle/11362/27858/ S1100447_es.pdf?sequence=1

- Sistema Económico Latinoamericano y del Caribe (SELA). (2011). Infraestructura física para la integración en América Latina y El Caribe. Caracas, Venezuela: Secretaría Permanente del SELA. Recuperado de http://walk. 
sela.org/attach/258/EDOCS/SRed/2011/06/T023600004771-0-Infraestructura_fisica_para_la_integracion_del_ALC.pdf

- Vassallo Magro, J. M. (2015). Asociación Público Privada en América Latina. Aprendiendo de la experiencia. Bogotá, Colombia: Corporación Andina de Fomento. Recuperado de http://scioteca.caf.com/handle/123456789/758

\section{Notas}

1 FMI, por sus iniciales.

2 IPEC, por sus iniciales.

3 OMC, por sus iniciales.

4 CEPAL, por sus iniciales. 